\title{
The effect of telehealth systems and satisfaction with health expenditure among patients with metabolic syndrome
}

\author{
Shu-Lin Uei ${ }^{\mathrm{a}}$, Chung-Hung Tsai ${ }^{\mathrm{b}}$ and Yu-Ming Kuo ${ }^{\mathrm{c}, *}$ \\ ${ }^{a}$ Department of Telecare, Mennonite Christian Hospital, Hualien, Taiwan \\ ${ }^{\mathrm{b}}$ Department of Health Administration, Tzu Chi University of Science and Technology, Hualien, Taiwan \\ ${ }^{\mathrm{c}}$ Department of Marketing and Distribution, Tzu Chi University of Science and Technology, Hualien, \\ Taiwan
}

\begin{abstract}
Telehealth cost analysis has become a crucial issue for governments in recent years. In this study, we examined cases of metabolic syndrome in Hualien County, Taiwan. This research adopted the framework proposed by Marchand to establish a study process. In addition, descriptive statistics, a $t$ test, analysis of variance, and regression analysis were employed to analyze 100 questionnaires. The results of the $t$ test revealed significant differences in medical health expenditure, number of clinical visits for medical treatment, average amount of time spent commuting to clinics, amount of time spent undergoing medical treatment, and average number of people accompanying patients to medical care facilities or assisting with other tasks in the past one month, indicating that offering telehealth care services can reduce health expenditure. The statistical analysis results revealed that customer satisfaction has a positive effect on reducing health expenditure. Therefore, this study proves that telehealth care systems can effectively reduce health expenditure and directly improve customer satisfaction with medical treatment.
\end{abstract}

Keywords: Telehealth system, telehealth expenditure, customer satisfaction, metabolic syndrome

\section{Introduction}

Approximately $80 \%$ of people aged 65 years or older in Taiwan have multiple chronic conditions, and the problems encountered in nursing elderly patients impact the home health care industry and social welfare system [1], telehealth care has been suggested as an effective approach to improving the health care system are resolving social problems. Telehealth care and medical care involve using information and communications technologies and electronic medical equipment to assist medical professionals in becoming more familiar with the community and home environments. Telecare allows older people to be cared for in their own family home environments, this has been shown to increase older people's autonomy and levels of self-esteem. This is a global trend developing in medical technology and services industries worldwide, and it is a policy currently being promoted by governments [2]. For example, since 2011, the i-Japan strategy has been focused on achieving health care reform through using telemedicine

\footnotetext{
${ }^{*}$ Corresponding author: Yu-Ming Kuo, Department of Marketing and Distribution, Tzu Chi College of Technology, 880, Sec. 2, Chien-Kuo Road, Hualien 97005, Taiwan. Tel.: +886 3857 2158; Fax: +886 3857 8941; E-mail: ss248@ tccn.edu.tw.
}

0928-7329/16/\$35.00 (C) 2016 - IOS Press and the authors. All rights reserved This article is published online with Open Access and distributed under the terms of the Creative Commons Attribution NonCommercial License. 
technology to maintain and enhance the medical expertise of physicians and to promote regional cooperation, with the objective of promoting and realizing the use of electronic health records and medical information in Japan to promote the use of electronic prescriptions. Thus, the Japanese telehealth care system has been developed to collect disparate health information and to integrate it with forecasting to benefit individuals and various health-related units.

Although telemedicine has been promoted in Taiwan for more than 10 years, many of the current plans have either been shut down or are economically ineffective, and other plans rely on government funds. May et al. [3] attributed this to the following four reasons: (1) blind pursuit of the latest advanced technologies; (2) network bandwidth costs; (3) legal and professional licensure restrictions; and (4) inadequacies associated with health insurance and government subsidies. In addition, from perspective of technology users, the diffusion of innovative telemedicine technologies is typically slow. Examples of barriers to implementing such systems include patients and customers who use telemedicine systems or might not know how to use them properly, and they might be concerned about their privacy [4]. Therefore, this study analyzed the health expenditure associated with telehealth care services in terms of health expenditure-effectiveness and patient satisfaction.

Telehealth costs analysis has become a crucial issue for governments and medical units in recent years. Thomas et al. [5] analyzed the long-term efficiency and cost-effectiveness of the telehealth care system introduced by the National Health Service (NHS) in the United Kingdom. That study involved a sample of 1,200 people who were involved in developing telehealth care policy for at least 12 months. The project included independent health management, optimization of treatment, such as coordination and patient care, to improve the NHS and other services. Rosenberg et al. [6] studied patient-centered medical care in a home setting to verify the UPMC telehealth care model. The study participants were patients who received such services between 2008 and 2010. The findings of that study showed that the model was effective in reducing medical and drug costs while improving the quality of health care services, particularly in reducing the cost of medical emergencies. Cryer et al. [7] studied a health care model employed by the New Mexico Family Hospital, and showed that patients who participated in a telemedicine program had a better health status and higher satisfaction compared with those who did not participate in the program. Moreover, the program reduced health care costs by $19 \%$. In a study involving patients with chronic conditions, Baker et al. [8] showed that telehealth care services can reduce medical costs by $7.7 \%-13.3 \%$, and recommended exploring the impact such systems have had on the field of telehealth care, as well as the benefits they provide in managing various diseases.

In this study, we examined cases involving patients with metabolic syndrome in Hualien County, Taiwan. In 2013, a preventative integrated community health screening service was conducted in three parts, and people over 40 years old were encouraged to undergo screening for high blood pressure, high blood sugar, and high cholesterol. Abnormally high results were observed in $59.42 \%$ of blood pressure tests, $19.18 \%$ of blood glucose tests, and $21.03 \%$ of blood lipid tests. Moreover, Hualien County is one of several counties representing the large gap between urban and rural areas; in particular, the traffic conditions are inconvenient, highlighting the importance of implementing telemedicine networks by integrating the use of medical equipment in home environments and building a business model for managing remote health care records to provide a more complete telehealth care service.

\section{Research methods}

In this study, we adopted the concept of reengineering based on the framework proposed by Marchand to assess individual cases, including routine care (monitoring of physiological measurement values, 
such as blood pressure and blood sugar, conducting daily telephone interviews, and monitoring incoming calls), emergency treatment, visits to outpatient, inpatient, and emergency departments, and health education, and used a structured questionnaire to obtain data measure the effectiveness of the platform based on the obtained benefits.

In this study, patients with metabolic syndrome were included if they met the following selection criteria: (1) systolic blood pressure $>130 \mathrm{~mm} \mathrm{Hg}$ or diastolic blood pressure $>85 \mathrm{~mm} \mathrm{Hg}$; (2) fasting plasma glucose $>100 \mathrm{~mm} \mathrm{Hg} / \mathrm{dl}$; (3) high-density lipoprotein cholesterol $(<40 \mathrm{mg} / \mathrm{dl}$ for men; $<50$ $\mathrm{mg} / \mathrm{dl}$ for women); (4) triglyceride $>150 \mathrm{mg} / \mathrm{dl}$; (5) body mass index $>25$; (6) at least 20 years of age; and (7) fluent in Chinese or Taiwanese.

This study used SPSS Version 22.0 for Windows for the analysis. The employed statistical methods were descriptive statistics, a $t$ test, analysis of variance, and regression analysis.

The following questionnaire was used:

A. Assessment of health expenditure

1. Please indicate whether the patient has any of the following diseases:
$\square$ Hypertension
$\square$ Diabetes
$\square$ Heart disease
$\square$ Stroke
$\square$ Other (Multiple items may be selected)

2. For the previous month, please estimate the following health expenditure:

(1) Out-of-pocket expenses: NT\$

(2) Total health care expenditure: NT\$

(3) Total care expenditure: NT\$

(4) Total expenditure of replacement therapy: NT\$

\section{B. Opportunity expenditure measure}

1. In the previous month, the patient has visited the clinic for treatment times.

2. For the previous month, please estimate the average number of hours that the patient has spent travelling to the clinic for treatment:

3. For the previous month, please estimate the average transportation expenditure for each visit to the clinic for medical treatment: NT\$

4. For the previous month, please estimate the number of hours the patient has spent waiting to receive medical treatment:

5. For the past month, please estimate the average number of people who have accompanied the patient to the clinic for medical care:

C. Satisfaction survey 


\begin{tabular}{|c|c|c|c|c|c|}
\hline Subject & Strongly disagree & Disagree & General & Agree & Strongly agree \\
\hline $\begin{array}{l}\text { 1. I am satisfied with the stability of telehealth care } \\
\text { instruments and equipment. }\end{array}$ & $\square$ & $\square$ & $\square$ & $\square$ & $\square$ \\
\hline $\begin{array}{l}\text { 2. I am satisfied with the telehealth care services I } \\
\text { have used. }\end{array}$ & $\square$ & $\square$ & $\square$ & $\square$ & $\square$ \\
\hline $\begin{array}{l}\text { 3. I would recommend telehealth care services to } \\
\text { other people. }\end{array}$ & $\square$ & $\square$ & $\square$ & $\square$ & $\square$ \\
\hline $\begin{array}{l}\text { 4. Overall, I am very satisfied the telehealth care } \\
\text { services I have used at home. }\end{array}$ & $\square$ & $\square$ & $\square$ & $\square$ & $\square$ \\
\hline $\begin{array}{l}\text { 5. Medical personnel have recommended that I use } \\
\text { telehealth care services. }\end{array}$ & $\square$ & $\square$ & $\square$ & $\square$ & $\square$ \\
\hline $\begin{array}{l}\text { 6. Medical personnel understand my needs related } \\
\text { to using telehealth care services. }\end{array}$ & $\square$ & $\square$ & $\square$ & $\square$ & $\square$ \\
\hline $\begin{array}{l}\text { 7. Medical personnel have a high level of confi- } \\
\text { dence regarding my use of telehealth care services. }\end{array}$ & $\square$ & $\square$ & $\square$ & $\square$ & $\square$ \\
\hline $\begin{array}{l}\text { 8. Medical personnel have encouraged me to use } \\
\text { telehealth care services. }\end{array}$ & $\square$ & $\square$ & $\square$ & $\square$ & $\square$ \\
\hline $\begin{array}{l}\text { 9. When I use telehealth care services to commu- } \\
\text { nicate with medical personnel, they are willing to } \\
\text { listen to me. }\end{array}$ & $\square$ & $\square$ & $\square$ & $\square$ & $\square$ \\
\hline $\begin{array}{l}\text { 10. Medical personnel want to know my ideas } \\
\text { about using telehealth care services. }\end{array}$ & $\square$ & $\square$ & $\square$ & $\square$ & $\square$ \\
\hline
\end{tabular}

Table 1

$t$ test results

\begin{tabular}{lcccc}
\hline & Pretest & Posttest & Pretest-posttest & Significant level \\
\hline Medical expenditure (NT\$). & 45.6 & 47.36 & -1.76 & \\
Medical health care expenditure (NT\$). & 258.5 & 172.1 & 86.3 & $p<0.01$ \\
Number of clinical visits for medical treatment. & 1.11 & 0.80 & 0.32 & $p<0.01$ \\
Average time (hours) commuting to clinics. & 0.42 & 0.30 & 0.12 & $p<0.05$ \\
Average expenditure (NT\$) of commuting to the clinic. & 20.42 & 13.7 & 6.72 & $p<0.01$ \\
Number of hours undergoing medical treatment. & 1.70 & 1.02 & 0.68 & $p<0.05$ \\
Average number of people accompanying patients for medical care. & 0.32 & 0.22 & 0.1 & \\
\hline
\end{tabular}

Note. $N=100$. All items in the table are based on estimates for the previous month.

\section{Results}

We distributed and collected 100 questionnaires, all of which were completed, yielding a response rate of $100 \%$. Three investigators collected data through telephone interview or by visiting the user's, this involved oral questioning by investigators and completion of the questionnaire. Professional training was given to all three investigators and this included training with the interviews protocol to ensure rigour in the study and to confirm that all data was correctly collected. Data collection took six months to complete. Regarding the participants' demographic characteristics, the average age was $65 \pm 4.5$ years, $55 \%$ were men, $95 \%$ were married, $48 \%$ had an educational attainment above high school, $60 \%$ were unemployed or retired, $68 \%$ had high blood pressure, and $90 \%$ had diabetes. The $t$ test results in Table 1 reveal significant differences in the medical health expenditure, number of clinical visits for medical treatment, average amount of time spent commuting to clinics, amount of time spent undergoing medical treatment, and the average number of people accompanying patients to medical care. It means telehealth systems really can reduce average 86.3 (NT\$) medical health care expenditure and average 0.12 hours commuting to clinics. 


\begin{tabular}{ll}
\multicolumn{2}{c}{ Table 2 } \\
\hline \multicolumn{2}{c}{ Satisfaction } \\
\hline Control variables & \\
Age & 0.068 \\
Health expenditure & $0.666^{*}$ \\
Max VIF & 1.232 \\
$t$ value & 5.745 \\
Adjusted $R^{2}$ & 0.414 \\
\hline Note: ${ }^{*} p<0.01$. &
\end{tabular}

Statistical results reveal that the participants' satisfaction had a positive effect on reducing health expenditure (Table 2). The $t$ value (5.745) and adjusted $R^{2}(0.414)$ indicate that the overall model exhibited acceptable explanatory power. The relationship between age and satisfaction was non-significant.

\section{Conclusion}

Previous studies investigating the health care expenditure of patients using telehealth systems have failed to account for patient satisfaction and willingness to use such systems, primarily because no medical records exist regarding these dispersed or physiological data for the most complete and most immediate record of integration. Through the use of cloud technologies, hospitals, health professionals, human inspection centers, organizations, and communities should be integrated to obtain more complete personal records to promote the use of health information systems and to improve the processes involved in disease diagnosis, which would be an invaluable reference for medical personnel, health promotion teams, and health care managers. Regarding the practical and theoretical contributions of this research, this study is the first to track medical records of specific medical conditions, and it presents a complete survey of the health expenditure associated with telehealth care systems, as well as the level of user satisfaction with such systems.

The empirical analysis revealed significant differences in the medical health care expenditure, number of clinical visits for medical treatment, average amount of time spent commuting to clinics, amount of time spent undergoing medical treatment, average number of people accompanying patients to medical care facilities or assisting them with other tasks, indicating that offering telehealth care services can reduce health care expenditure. Among these results, reducing the expenditure of medical expenses can increase the amount of money patients have to cover their out-of-pocket expenses, which could allow them to purchase more effective drugs for treatment. Therefore, this study proves that telehealth care systems can effectively reduce health care expenditure and directly improve customer satisfaction for medical treatment.

\section{Research limitations}

This study encountered several limitations in the conceptualization and expansion of the theoretical model. Because our samples comprised patients residing in rural areas in Eastern Taiwan, the cases could not be monitored continuously, the sample was small, and the size of the area impeded the distribution of questionnaires. Another potential limitation is the restricted time and space the patients had when using telecare. However, this study does provide valuable and unique insights regarding the use of telecare services. 


\section{Acknowledgments}

This research was supported by grants from Mennonite Christian Hospital. The authors thank the participants in the community for their assistance in collecting the data for this study.

\section{References}

[1] Levy, S., Jack, N., Bradley, D., Morison, M., Swanston, M. Perspectives on telecare: The client view. J. Telemed. Telecare, 2003, 156-160.

[2] Wang, J.Y., Tsai, C.H., \& Wang, S.W. Using telecare system to construct medication safety mechanisms for remote area elderl. Journal of Chemical and Pharmaceutical Research, 2013, 1-5.

[3] May, C., Mair, F.S., Finch, T., MacFarlane, A., Dowrick, C., Treweek, S., Rapley, T., Ballini, L., Ong, B.N., Rogers, A., et al. Development of a theory of implementation and integration: Normalization process theory. Implement., 2009, 4-29.

[4] Sanders, J.H., \& Bashshur, R.L. Challenges to the implementation of telemedicine. Telemedicine Journal, 1995, 115-123.

[5] Thomas, C.L., Man, M.S., O'Cathain, A., Hollinghurst, S., Large, S., Edwards, L., Salisbury, C. Effectiveness and costeffectiveness of a telehealth intervention to support the management of long-term conditions: Study protocol for two linked randomized controlled trials. Trials, 2014, 15-36.

[6] Rosenberg, C.N., Peele, P., Keyser, D., McAnallen, S., \& Holder, D. Results From A Patient-Centered Medical Home Pilot At UPMC Health Plan Hold Lessons For Broader Adoption Of The Model. Health Affairs, 2012, 2423-2431.

[7] Cryer, L., Shannon, S.B., Amsterdam, M.V., \& Leff, B. Costs For 'Hospital At Home' Patients Were 19 Percent Lower, With Equal Or Better Outcomes Compared To Similar Inpatients. Health Affairs, 2012, 1237-1243.

[8] Baker, L.C., Johnson, S.J., Macaulay, D., \& Birnbaum, H. Integrated Telehealth and Care Management Program for Medicare Beneficiaries with Chronic Disease Linked To Savings. Health Affairs, 2011, 1689-1697. 